\title{
A Benchmarking and Sensitivity Study of the Full Two-Body Gravitational Dynamics of the DART Mission Target, Binary Asteroid 65803 Didymos
}

\author{
Harrison F. Agrusa ${ }^{\mathrm{a}, *}$, Derek C. Richardson ${ }^{\mathrm{a}}$, Alex B. Davis ${ }^{\mathrm{b}}$, Eugene Fahnestock ${ }^{\mathrm{c}}$, Masatoshi \\ Hirabayashi $^{\mathrm{d}}$, Nancy L. Chabot ${ }^{\mathrm{e}}$, Andrew F. Cheng ${ }^{\mathrm{e}}$, Andrew S. Rivkin ${ }^{\mathrm{e}}$, Patrick Michel ${ }^{\mathrm{f}}$, and the \\ DART Dynamics Working Group \\ ${ }^{a}$ Department of Astronomy, University of Maryland, College Park, MD 20742, USA \\ ${ }^{b}$ University of Colorado Boulder, 429 UCB, Boulder, CO 80309, USA \\ ${ }^{c}$ Jet Propulsion Laboratory, California Institute of Technology, Pasadena, CA 91109, USA \\ ${ }^{d}$ Auburn University, 39 Davis Hall, Auburn, AL 36849, USA \\ e Johns Hopkins University Applied Physics Laboratory, Laurel MD, 20723, USA \\ ${ }^{f}$ Universit Cte dAzur, Observatoire de la Cte dAzur, CNRS, Laboratoire Lagrange, 06304 Nice, France
}

\begin{abstract}
NASA's Double Asteroid Redirection Test (DART) is designed to be the first demonstration of a kinetic impactor for planetary defense against a small-body impact hazard. The target is the smaller component of the binary asteroid 65803 Didymos. We have conducted high-fidelity rigid full two-body simulations of the mutual dynamics of this system in a broad benchmarking exercise to find the best simulation methodologies, and to understand the sensitivity of the system to initial conditions. Due to the non-spherical shapes of the components and their close proximity, the components cannot be treated as point masses and so the dynamics differ significantly from a simple Keplerian orbit, necessitating the use of numerical simulations to fully capture the system's dynamics. We find that the orbit phase (angular position or true anomaly) of the secondary is highly sensitive to the initial rotation phase of the primary, making prediction of the secondary's location from numerical simulation challenging. Finally, we show that the DART impact should induce significant free and forced librations on the secondary. If this libration can be measured by ESA's recently approved follow-up spacecraft, Hera, it may be possible to constrain properties of the secondary's interior structure.

Keywords: Asteroids, dynamics, Rotational dynamics, Celestial mechanics, Near-Earth objects
\end{abstract}




\section{Introduction}

The Asteroid Impact \& Deflection Assessment (AIDA) collaboration is a NASA- and ESAsupported effort to test the capability of a kinetic impactor for hazardous asteroid mitigation. NASA will lead the Double Asteroid Redirection Test (DART) mission, which will achieve a kinetic impact on the secondary (Didymos B) of 65803 Didymos, a near-Earth binary asteroid, in the fall of 2022 (Cheng et al., 2018). ESA will lead Hera, a follow-up mission to rendezvous with Didymos to characterize the system and visible effects of the impact (Michel et al., 2018). The main goal of the DART mission is to demonstrate the kinetic impactor technique by intercepting the secondary, causing a change in the binary orbital period that can be measured with ground-based observations.

In this work, we present results from a suite of benchmarking simulations conducted by the DART Investigation Team's Dynamics Working Group to better understand the complex mutual dynamics, to constrain the sensitivity of the simulated Didymos system to initial conditions, and to identify the appropriate numerical methods to fully capture the dynamics. In Section 1.1 we describe the physical and dynamical properties of the Didymos system. Section 2 introduces the four dynamics models used in this study and the initial conditions of the simulation cases. The results are presented in Section 3. Finally, we discuss future work to better understand the Didymos binary in Section 4.

\subsection{The Didymos System}

Discovered in 1996, 65803 Didymos is classified as a near-Earth object and potentially hazardous asteroid with a heliocentric semi-major axis of 1.644 au (Alday et al., 1996). In 2003, it was discovered that Didymos is a binary system (Pravec et al., 2003). The binary has a mutual orbit period of $P_{\text {orb }} \sim 11.9217 \mathrm{~h}$ and a semi-major axis of $a_{\text {orb }} \sim 1.19 \mathrm{~km}$ (Pravec et al., 2006; Naidu et al., 2020a). According to the binary mean separation and orbital period, Kepler's third law for assumed point masses implies a system mass of $M_{\mathrm{sys}} \sim 5.37 \times 10^{11} \mathrm{~kg}$.

\footnotetext{
${ }^{*}$ Corresponding author

Email address: hagrusa@astro.umd. edu (Harrison F. Agrusa)
} 
The primary (Didymos A) is $\sim 780 \mathrm{~m}$ across and has an oblate shape and equatorial ridge, and the secondary (Didymos B) is approximately $164 \mathrm{~m}$ across and we assume an ellipsoidal shape similar to that of other NEO binary secondaries. Didymos A is a fast rotator, with a spin period of $2.26 \mathrm{~h}$. A polyhedral shape model with 1996 facets was derived by Naidu et al. (2020a) from combined radar and light curve data. In the simulations presented here, Didymos B is assumed to be synchronous (i.e., tidally locked) with its long axis initially aligned with the line of centers. It is also assumed that both bodies are in principal axis rotation and that their spin poles are initially aligned with the binary orbit normal.

Table 1 summarizes the relevant physical and dynamical parameters of the Didymos system. These are the nominal system parameters adopted by the DART investigation team at the current time and will be updated throughout the DART mission as new measurements become available.

\section{Methodology}

The Didymos binary is an example of the full two-body problem (F2BP), where the rotational and translational dynamics are fully coupled, due to the objects' irregular shapes and the close proximity of the components. As a result, the system's dynamical evolution is especially sensitive to the shapes and initial positions and orientations of each component, thus F2BP simulation codes are necessary to fully capture the system's dynamics.

\subsection{The Simulation Codes}

Four different codes were tested in this study, each developed by team members at NASA JPL, University of Colorado Boulder (UCB), Auburn University, and the University of Maryland (UMd), respectively. Only some of these codes have official names, so we refer to each code by the institution that developed it for simplicity. Brief descriptions of the codes are given below.

NASA JPL. The JPL code is based on the formulation of the mutual gravitational potential between two polyhedral bodies developed by Werner and Scheeres (2005). It calculates the mutual gravitational potential and its gradients through a Legendre polynomial series expansion, truncated to a desired order, and integrates the discrete-time Hamiltonian equations of motion using the LieGroup Variational Integrator (LGVI) developed by Lee et al. (2007). This code was written in C++ 


\begin{tabular}{|c|c|c|c|}
\hline Symbol & Parameter & Value & Comments/References \\
\hline$a_{\mathrm{orb}}$ & Semi-major Axis & $1.19 \pm 0.03 \mathrm{~km}$ & (Naidu et al., 2020a) \\
\hline$c: b: a$ & Secondary Axis Ratios & $1: 1.2: 1.56$ & Assumed, based on other binary systems. \\
\hline$D_{\mathrm{P}}$ & Diameter of Primary & $780 \pm 30 \mathrm{~m}$ & (Naidu et al., 2020a) \\
\hline$D_{\mathrm{S}}$ & Diameter of Secondary & $164 \pm 18 \mathrm{~m}$ & Derived from $D_{\mathrm{P}}$ and $D_{\mathrm{S}} / D_{\mathrm{P}}$ \\
\hline$D_{\mathrm{S}} / D_{\mathrm{P}}$ & Size Ratio & $0.21 \pm 0.01$ & (Scheirich and Pravec, 2009) \\
\hline$e_{\text {orb }}$ & $\begin{array}{l}\text { Binary Orbit } \\
\text { Eccentricity }\end{array}$ & $e_{\text {orb }}<0.03$ & $\begin{array}{l}\text { Upper limit, assumed zero. (Scheirich and } \\
\text { Pravec, 2009) }\end{array}$ \\
\hline$i_{\text {orb }}$ & $\begin{array}{l}\text { Binary Orbit } \\
\text { Inclination }\end{array}$ & 0.0 & Assumed. \\
\hline$(\lambda, \beta)$ & Mutual Orbit Pole & $\left(310^{\circ},-84^{\circ}\right) \pm 10^{\circ}$ & $\begin{array}{c}\text { Ecliptic coordinates, (Scheirich and Pravec, } \\
\text { 2009; Naidu et al., 2020a) }\end{array}$ \\
\hline$M_{\text {sys }}$ & Total System Mass & $(5.37 \pm 0.44) \times 10^{11} \mathrm{~kg}$ & $\begin{array}{c}\text { Derived via Kepler's 3rd Law with } P_{\text {orb }} \text { and } \\
\qquad a_{\text {orb }} .\end{array}$ \\
\hline$P_{\text {orb }}$ & Binary Orbit Period & $11.9217 \pm 0.0002 \mathrm{~h}$ & $\begin{array}{l}\text { One possible orbit solution. (Scheirich and } \\
\text { Pravec, 2009) }\end{array}$ \\
\hline$P_{\mathrm{P}}$ & Primary Spin Period & $2.2600 \pm 0.0001 \mathrm{~h}$ & (Pravec et al., 2006) \\
\hline$P_{\mathrm{S}}$ & Secondary Spin Period & $11.9217 \mathrm{~h}$ & Assumed. \\
\hline$\rho_{\mathrm{P}}$ & Primary Bulk Density & $2170 \pm 350 \mathrm{~kg} \mathrm{~m}^{-3}$ & Derived based on $D_{\mathrm{P}}$ and $M_{\text {sys }}$. \\
\hline$\rho_{\mathrm{S}}$ & $\begin{array}{l}\text { Secondary Bulk } \\
\text { Density }\end{array}$ & $2170 \pm 350 \mathrm{~kg} \mathrm{~m}^{-3}$ & Assumed. \\
\hline
\end{tabular}

Table 1: Physical and dynamical parameters of the Didymos System. These are the current nominal values adopted by the DART investigation team. Because these parameters are constantly being refined by ongoing observations, these are not exactly the same parameters used in this study. The initial conditions of the simulations presented here differ slightly, but remain within the uncertainty bounds given here. (See Table 3 for the simulation initial conditions.) 
and parallelized to run on a cluster computer environment, due to the high computational cost of the potential and gradients evaluation at each timestep.

University of Colorado Boulder (UCB). The recently developed UCB code utilizes inertia integrals to expand the mutual gravitational potential according to the formalism derived by Hou et al. (2017). This tool, known as the General Use Binary Asteroid Simulator (GUBAS), is now publicly available $^{1}$ and can easily be run on a single desktop computer. Despite the different mathematical formulations for the mutual gravitational potential and its gradients between the JPL and UCB codes, they agree to near-machine precision for the same given expansion order of the mutual potential, since they used the same numerical integrator (LGVI) for the simulations run herein. However, the inertia integral formulation allows for the attitude and mass distribution to be decoupled and computed separately, which allows for a more computationally efficient implementation and thus faster runtimes. The present study served as a convenient test to confirm that the UCB code does in fact achieve the same result as the JPL code.

Auburn University. The Auburn code is a simplified version of the UCB code. It expands the inertia integrals only to second order according to the formulation given by Hirabayashi and Scheeres (2013). The equations of motion are solved with an 8th-order Runge-Kutta scheme. This code can be thought of as evaluating the mutual gravitational potential of the system as if the Didymos A shape model were replaced with a best-fit ellipsoid. Although the Auburn code does not fully capture perturbations due to the asymmetric shape of the primary, it is extremely fast and is a useful reference point to understand the effect of higher-order perturbations due to Didymos A's shape.

University of Maryland (UMd). Unlike the other three codes that represent the primary and secondary as monolithic and homogeneous bodies of some arbitrary polyhedral or ellipsoidal shape, the UMd code treats each body as a rigid aggregate of many spherical particles. The code, called PKDGRAV, is a parallel $N$-body tree code (Richardson et al., 2000; Stadel, 2001). The UMd code uses a primary consisting of $\sim 3500$ particles in order for the average particle diameter $(\sim 42 \mathrm{~m})$ to

\footnotetext{
${ }^{1}$ https://github.com/alex-b-davis/gubas
} 


\begin{tabular}{ccc} 
& Primary & Secondary \\
\hline$N$ & 3355 & 3546 \\
$\rho_{\text {bulk }}\left[\mathrm{g} \mathrm{cm}^{-3}\right]$ & 2.104 & 2.104 \\
$\rho_{\text {particle }}\left[\mathrm{g} \mathrm{cm}^{-3}\right]$ & 3.8947 & 3.7329 \\
$r_{\text {avg }}[\mathrm{m}]$ & 21 & 4.4
\end{tabular}

Table 2: Physical parameters for pkdgrav particles. All particles in each respective body are given a uniform particle density in order to achieve the desired bulk density of $2.104 \mathrm{~g} \mathrm{~cm}^{-3} \cdot r_{\text {avg }}$ is the mean particle radius. The particle size distributions for the primary and secondary are sampled from a normal distribution with mean $\mu=21 \mathrm{~m}$, standard deviation $\sigma=4.2 \mathrm{~m}$ and $\mu=4.4 \mathrm{~m}, \sigma=0.88 \mathrm{~m}$, respectively. The size distributions both have $\pm 1 \sigma$ cutoffs.

be within the spatial resolution of the radar shape model $(\sim 50 \mathrm{~m})$. Details of UMd's representation of each body are shown in Table 2. The translational motion is integrated with a fixed-step second-order leapfrog integrator, while the rotational motion is integrated with a time-adaptive fourth-order Runge-Kutta scheme within each leapfrog step (Richardson et al., 2009). Note that PKDGRAV's k-d tree is not used, so the forces and torques are computed by summing over every particle at every timestep to ensure the highest possible accuracy at the expense of speed.

Snapshots of the simulations of Didymos are shown in Fig. 1. The key differences are that JPL, UCB, and Auburn simulate the full radar-derived Didymos A shape model with an ellipsoidal Didymos B, with the mutual gravitational potential expanded to some desired order of accuracy. The UMd method fills a volume with randomly packed spherical particles, then carves each body to match the desired shape and computes the mutual gravitational potential of the packed spheres exactly without truncation to any order. Because of the different mass representations of each method, there will be inherent variations between these approaches.

\subsection{Initial Conditions}

The 11 simulations presented here comprise a small subset of cases that the Dynamics Working Group has studied thus far. These simulations were selected to compare code performance and better understand the system's sensitivity to uncertainty in its initial state. Using the nominal values for the mass of each body and their separation, we computed the initial conditions to approximately put the system on a circular Keplerian orbit. This is considered the "nominal" simulation case. More details on the initial conditions for the nominal case are shown in Table 3. We then give these initial conditions slight perturbations to test the system's sensitivity to the 


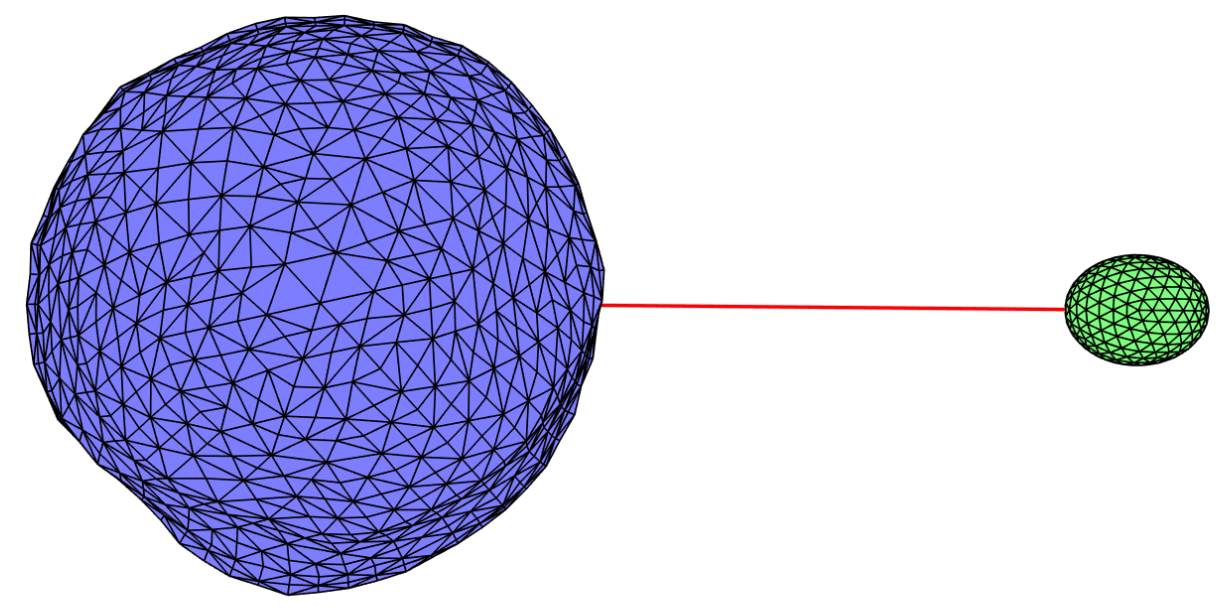

(a) Radar shape model of Didymos A and the assumed ellipsoidal shape of Didymos B. JPL, UCB, and Auburn simulate the full radar-derived primary shape model with the mutual potential expanded to various orders of accuracy.
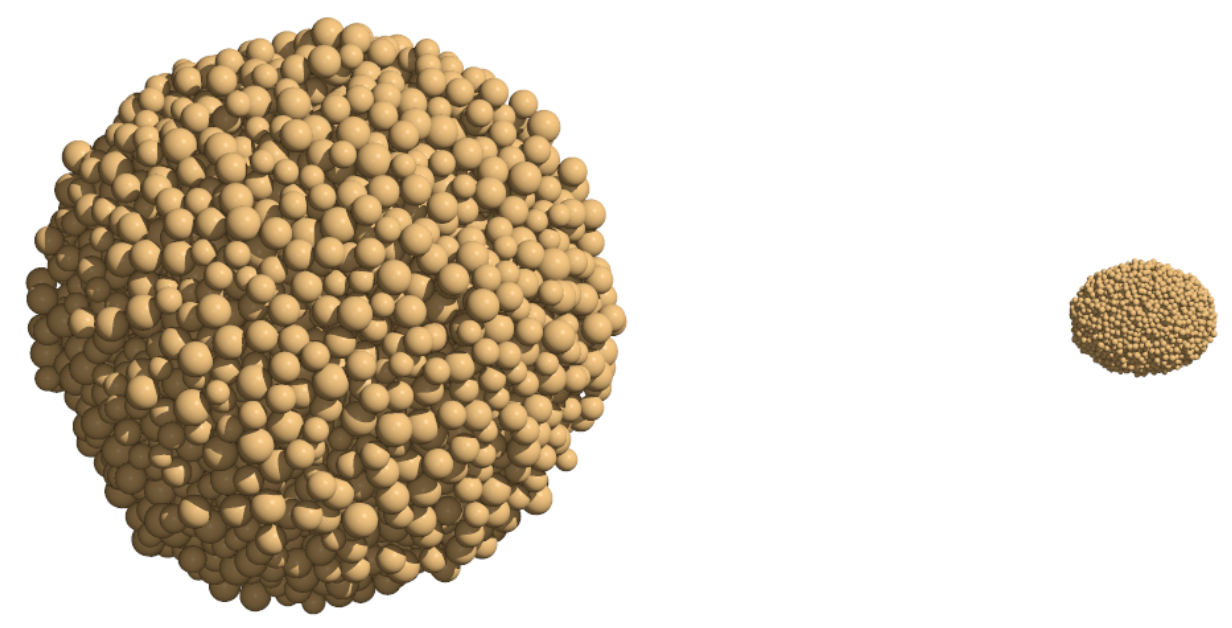

(b) The UMd representation of the binary, where randomly packed spherical particles fill the shapes of each body and the potential is computed explicitly over every particle.

Figure 1: Representations of the Didymos binary among the different codes. Both of these images are a top-down view (i.e. from the mutual orbit north pole) at the start of the simulation. 
initial relative velocity of the secondary and the initial rotation phase of the primary. See Table 4 for details of the 11 test cases, along with a schematic in Fig. 2. Each group selected a timestep for their respective code, based on numerical convergence and runtime constraints, with each code conserving energy to one part in a million or better over the entire simulation. Each of the 11 test cases was simulated for a total of 150 days of simulation time. All codes modeled the system as two rigid bodies interacting purely through their mutual gravity, with all additional forces or torques such as solar tides, BYORP, or internal dissipation turned off.

Each group output previously agreed-upon state variables at a 1-minute cadence (except for UMd which had hourly outputs due to data storage constraints). With such a high output cadence, we were able to compare both short- and long-term evolution of the binary system with each simulation code. The JPL and UCB codes were run with the mutual gravity expansion set to 4th order; this choice is accurate enough to capture the dynamics with high fidelity while keeping the computation time manageable. UCB also repeated the nominal case with the gravity expansion order set to 8th order to confirm that the choice of 4th order was indeed sufficient to accurately model the system. Again, the Auburn code is limited to 2nd order, while the UMd code has no order truncation.

\section{Results}

\subsection{Code Performance}

Since each code was run on different machines (see acknowledgments) with different timesteps and numerical routines, normalized performance comparisons can be troublesome. In Table 5, we simply show the runtimes for the nominal case along with the number of processors and timesteps used by each code. The Auburn code is orders of magnitude faster than the other codes, given its 2nd-order approximation of the mutual potential. This makes it a useful tool for quick tests, however it does not capture higher-order perturbations due to the asymmetric shape of the primary. It should also be noted that the UCB or JPL codes would have similar performance if the mutual potential approximation were set to 2 nd order. The UMd code had the longest runtime, due to a combination of its small timestep and requirement to compute the gravitational potential on 


\begin{tabular}{ccc} 
Parameter & Value & Notes \\
\hline System Mass & $5.276428 \times 10^{11} \mathrm{~kg}$ & \\
\hline Primary Mass & $5.228011 \times 10^{11} \mathrm{~kg}$ & \\
\hline Secondary Mass & $4.841661 \times 10^{9} \mathrm{~kg}$ & \\
\hline Primary Bulk Density & $2103.4 \mathrm{~kg} \mathrm{~m}^{-3}$ & \\
\hline Secondary Bulk Density & $2103.4 \mathrm{~kg} \mathrm{~m}^{-3}$ & $a$ is oriented along line of centers at $t=0$. \\
\hline Secondary Axis Lengths & $\begin{array}{c}a=103.16 \mathrm{~m} \\
b=79.35 \mathrm{~m} \\
c=66.13 \mathrm{~m}\end{array}$ & \\
\hline Initial Body Separation & $1.18 \mathrm{~km}^{-1}$ & $\begin{array}{c}\text { Relative velocity of the body centers. Derived to achieve } \\
\text { circular Keplerian orbit with period of 11.9216 h. }\end{array}$ \\
\hline Initial Relative Velocity & $0.17275 \mathrm{~m} \mathrm{~s}^{-1}$ & $\begin{array}{c}\text { Equivalent to a 2.26 h spin period. Aligned with mutual } \\
\text { orbit pole. }\end{array}$ \\
\hline $\begin{array}{c}\text { Primary Spin Angular } \\
\text { Velocity }\end{array}$ & $0.0007723 \mathrm{rad} \mathrm{s}^{-1}$ & $\begin{array}{c}\text { Equivalent to a spin period of 11.9216 h, in order to match } \\
\text { the Keplerian orbit period. Aligned with orbit pole. }\end{array}$ \\
\hline $\begin{array}{c}\text { Secondary Spin Angular } \\
\text { Velocity }\end{array}$ & $0.0001464 \mathrm{rad} \mathrm{s}^{-1}$ &
\end{tabular}

Table 3: Initial conditions of the nominal simulation.

\begin{tabular}{|c|c|}
\hline Name & Description \\
\hline nominal & $\begin{array}{l}\text { The nominal, unperturbed initial state. The initial conditions are calculated using Newton's } \\
\text { version of Kepler's Third Law to give a circular orbit based on the total system mass and } \\
\text { mean separation. }\end{array}$ \\
\hline posR, negR & $\begin{array}{l}\text { The initial velocity of the secondary’s barycenter is perturbed by } \pm 0.0005 \mathrm{~m} / \mathrm{s} \text { in the } \\
\text { instantaneous orbital radial direction. }\end{array}$ \\
\hline posT, negT & $\begin{array}{l}\text { The initial velocity of the secondary’s barycenter is perturbed by } \pm 0.0005 \mathrm{~m} / \mathrm{s} \text { in the } \\
\text { instantaneous orbital tangential direction (along-track). }\end{array}$ \\
\hline posN, negN & $\begin{array}{l}\text { The initial velocity of the secondary’s barycenter is perturbed by } \pm 0.0005 \mathrm{~m} / \mathrm{s} \text { in the } \\
\text { instantaneous orbital normal direction (out-of-plane). }\end{array}$ \\
\hline $\begin{array}{r}\mathrm{ph}+1, \mathrm{ph}+3 \\
\mathrm{ph}-1, \mathrm{ph}-3\end{array}$ & $\begin{array}{c}\text { the initial rotation phase of primary shape model is adjusted by rotating } \pm 1 \text { or } \pm 3 \text { degrees } \\
\text { from nominal, around the primary spin pole. }\end{array}$ \\
\hline
\end{tabular}

Table 4: Description of the 11 simulation cases. 


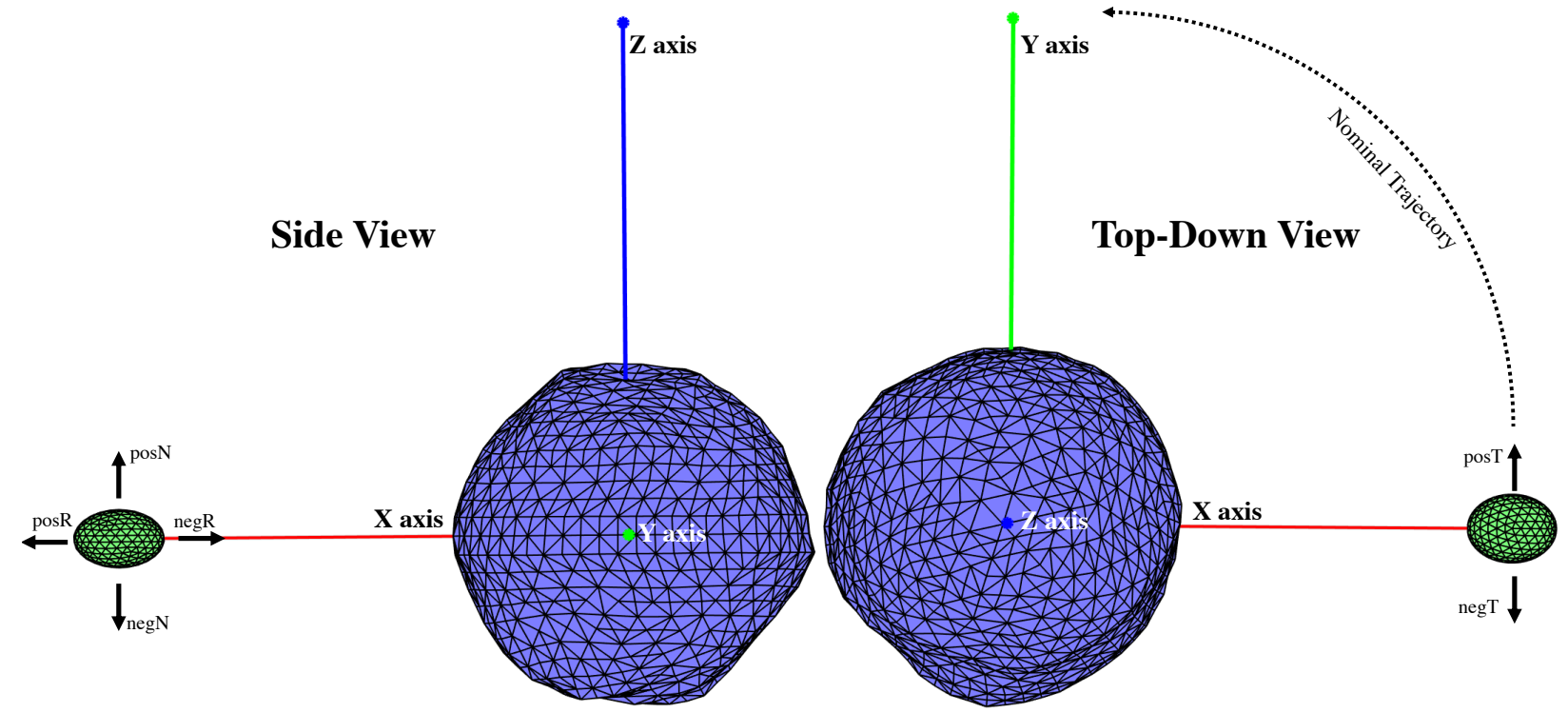

Figure 2: Schematic of the perturbations to Didymos B. The X, Y, and Z axes are the three principal axes of Didymos A. The nominal case has the long axes of both bodies aligned. The $\mathrm{ph} \pm 3$ and $\mathrm{ph} \pm 1$ cases have Didymos A rotated about its spin axis such that its $\mathrm{X}$ axis is pointed \pm 3 or \pm 1 degrees away from the direction to Didymos $\mathrm{B}$.

\begin{tabular}{ccccc} 
& \# Processors & timestep [s] & \# timesteps & wallclock [h] \\
\hline Auburn & 1 & 60 & 216,000 & $\sim$ several min \\
NASA JPL (4th Order) & 512 & 40 & 324,000 & 38.0 \\
UCB (4th Order) & 1 & 40 & 324,000 & 5.6 \\
UCB (8th Order) & 1 & 40 & 324,000 & 111.55 \\
UMd & 4 & 1.875 & $6,912,000$ & 702.0
\end{tabular}

Table 5: Each code's performance for its nominal run, with a total integration duration of $150 \mathrm{~d}$.

a particle-by-particle basis. The 4th-order UCB code offers the best combination of speed and accuracy, as we will see in the following section. Therefore, the Dynamics Working Group has recommended that the UCB code be adopted for future rigid-body dynamics studies related to DART.

\subsection{The Nominal Case}

The orbit period, semi-major axis, and eccentricity for each code's nominal case is shown in Table 6 along with what those values would be if the system were Keplerian. As expected, the NASA JPL and UCB (4th Order) results are nearly identical. They also match closely to the 8th-order result, indicating that the 4th-order approximation is capturing the mutual gravitational 


\begin{tabular}{cccc} 
& Orbit Period $[\mathrm{h}]$ & Semi-Major Axis $[\mathrm{km}]$ & Eccentricity \\
\hline Auburn & 11.8138797 & 1.1747401 & 0.0055961390 \\
NASA JPL & 11.8062721 & 1.1743513 & 0.0059765762 \\
UCB (4th Order) & 11.8062824 & 1.1743520 & 0.0059765772 \\
UCB (8th Order) & 11.8053794 & 1.1743062 & 0.0060210983 \\
UMd & 11.8211246 & 1.1750370 & 0.0051450228 \\
Kepler Orbit & 11.9216030 & 1.1800000 & 0.0000000000
\end{tabular}

Table 6: Simulated time-averaged orbital period, semi-major axis, and eccentricity for the nominal case. All codes use exactly the same initial conditions and body masses, so the deviations from a Keplerian orbit and among the codes themselves are due to different mass representations of the primary and secondary. Each quantity is rounded to enough decimal points to show deviation between nearly identical numbers.

potential with high fidelity. The deviations in the orbit period and semi-major axis are driven by each code's representation of the mass distribution, and thus the mutual potential, of the two bodies.

Figure 3 shows the evolution of the system over $2 \mathrm{~d}$ ( $\sim 4$ orbital periods) as determined by each simulation code for the nominal case. Since the bodies do not follow a precise Keplerian orbit due to their irregular shapes, the orbital eccentricity and inclination are osculating-they are instantaneous values evaluated based on the position and motion of the body centers for each simulation output.

Since each group used a numerically converged timestep, the differences they show in the system's orbital evolution are attributable to how each code represents the mass distribution of each body. The oscillations in the various orbital elements are small and are driven by the shape perturbations of the primary. The bottom two plots in Fig. 3 show the obliquities of each body, defined as the angle between the body's spin axis and the mutual orbit pole. Due to its 2nd-order gravity approximation, the Auburn code shows negligible out-of-plane motion, indicating that the small changes in the inclination and obliquities with the other three codes are mainly driven by asymmetries in the primary shape.

The NASA JPL and UCB codes at 4th order are indistinguishable in Fig. 3 and the 8th-order version shows almost no appreciable difference. All codes show qualitative agreement, given their known differences. Because the UMd code had an output frequency of one hour, the inclination and obliquity plots look artificially jagged. 

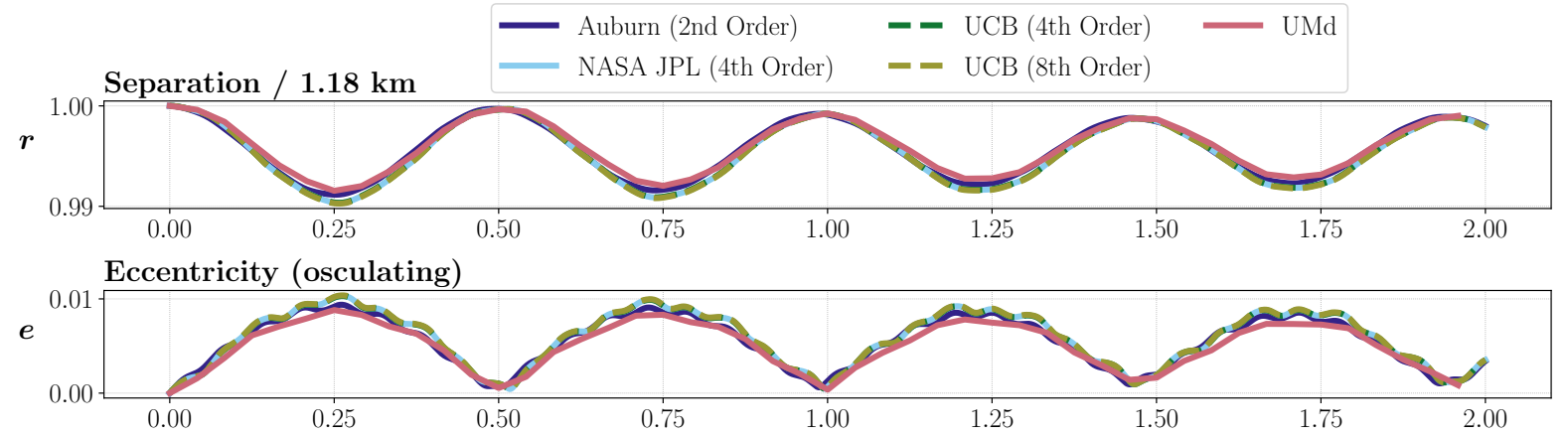

Inclination (osculating), degrees

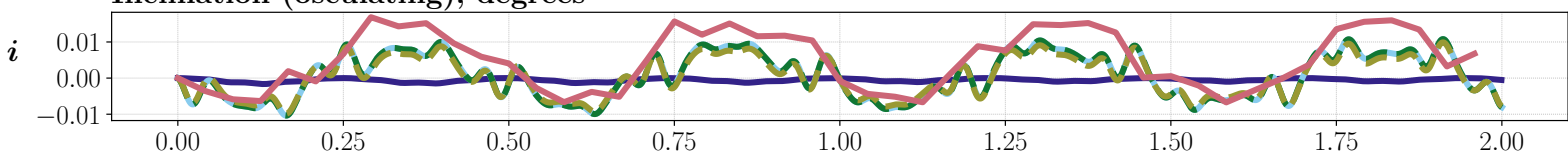

Didymos A Obliquity, degrees

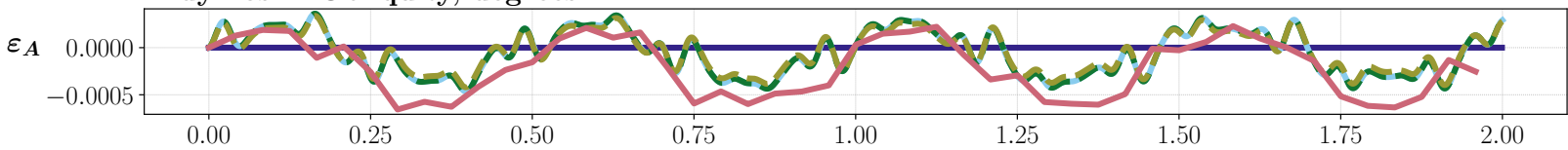

Didymos B Obliquity, degrees

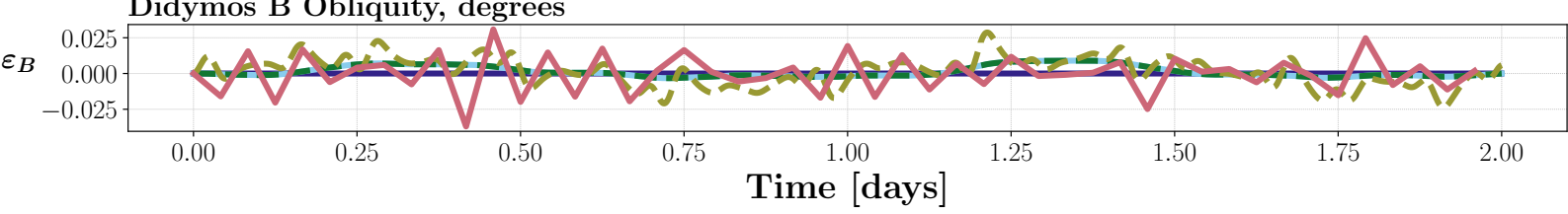

Figure 3: The nominal case over $2 \mathrm{~d}$ ( $\sim 4$ orbital periods). The NASA JPL and UCB codes show perfect agreement at 4 th order, and the UCB 8th-order version matches closely as well. The Auburn code is evaluating the mutual gravitational potential to 2nd order, so it doesn't capture higher-order effects of the primary's asymmetric shape on the mutual orbit. The choppy noise in UMd's plot of Didymos B's Obliquity is a result of its coarser output cadence. In order to distinguish each curve, this figure is best viewed in the online color version. 

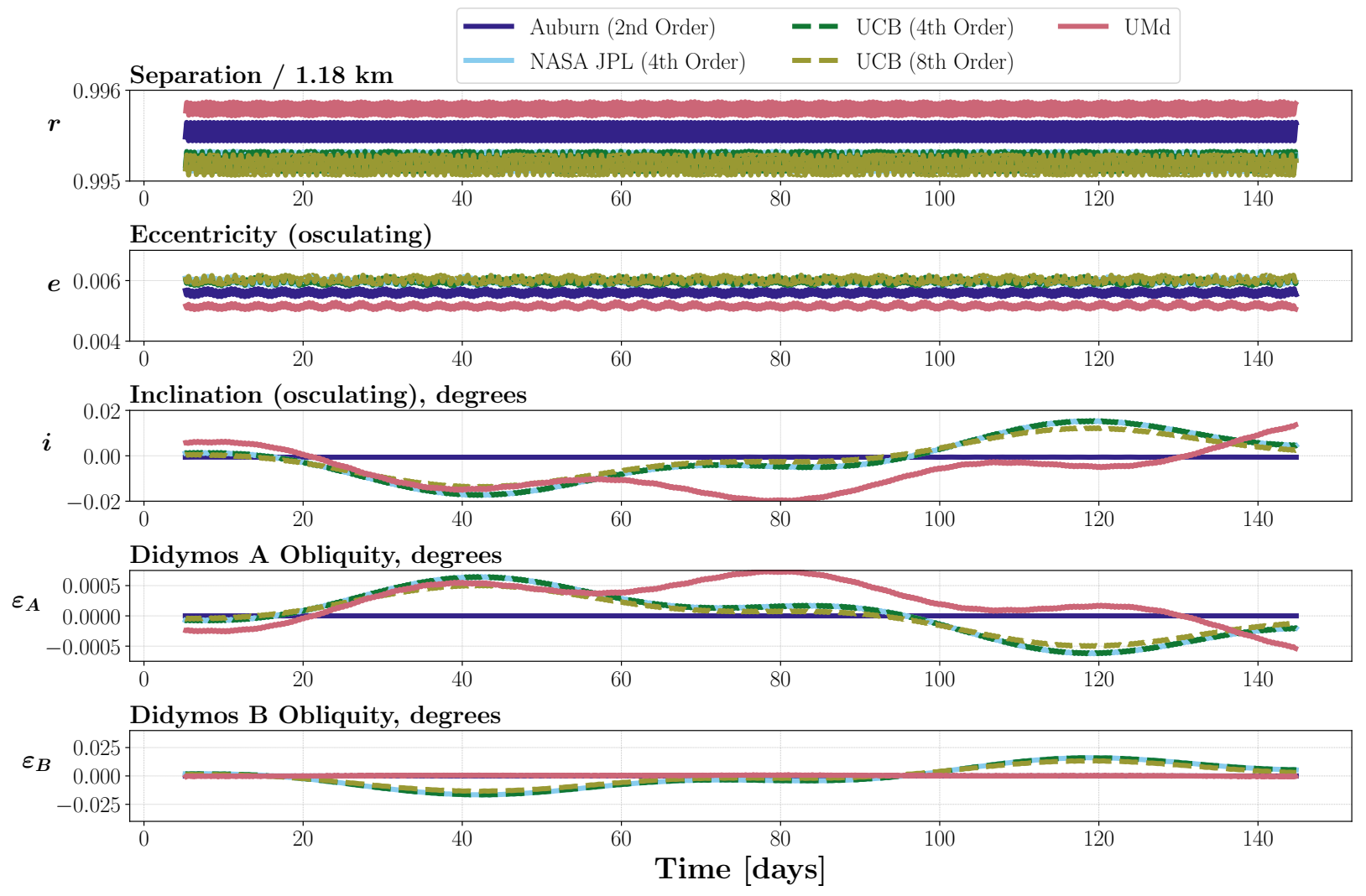

Figure 4: A running average of orbit parameters for the nominal case over 5 months. The first and last $10 \mathrm{~d}$ of data are cut off due to the running average using $\sim 10$ days worth of data (20 orbital periods). In order to distinguish each curve, this figure is best viewed in the online color version.

To study the orbital elements over longer time scales, we take a running average to remove short-term behavior. This is shown for the nominal case over the full 5-month simulation in Fig. 4. The long-term evolution is quite stable, with the orbital elements remaining constant or drifting by a very small amount. One apparent effect is that each code has a different average semimajor axis and eccentricity. This is again a result of different mass representations, which sets the initial mutual potential, and thus the eccentricity and equilibrium separation. This highlights the influence of the primary's shape on the orbital properties of the system. In general, the codes agree well, with differences attributable to their respective representations of each body. Because we expect the Didymos binary to be stable over long periods, our ability to capture long-term stability in its mutual orbit is reassuring. 


\subsection{Primary Rotation Phase}

The only constraints we place on the orientations of the primary and secondary are that their spin axes be initially aligned with the mutual orbit pole. In our nominal case, the primary's long axis is aligned with the line of centers at $t=0$, but this choice is arbitrary. A precise measurement of the primary's orientation relative to the secondary at a given epoch with ground-based observations prior to the DART impact will be very challenging, so we treat the primary's initial rotation phase as a free parameter. Therefore, understanding the system's sensitivity to the initial primary rotation phase is essential to developing methods for predicting the position of the secondary at later times.

To test this sensitivity, we varied the initial primary rotation phase with respect to the nominal case by $\pm 3^{\circ}$ and $\pm 1^{\circ}$. All codes showed a non-negligible dependence on this slight change. Fig. 5 shows the orbital phase (angular position of secondary) relative to each code's respective nominal case. The Auburn code is comparatively insensitive to the initial primary phase since it is only approximating the mass distribution to second order. NASA JPL and UCB have identical results, and UMd is slightly more sensitive to the initial primary phase.

If the initial primary rotation phase is altered, the initial mass distribution will be slightly different, resulting in a different mutual potential and thus a different orbital period. Due to the asymmetry of the primary and the binary's small separation, this is a non-negligible effect, especially if we want to accurately predict the position of the secondary.

After determining that the orbit may be sensitive to the initial primary rotation phase, we performed another set of simulations over a wider range of initial rotation phases with the UMd code only. The results in Fig. 6 show that the initial primary rotation phase has a significant influence on the orbital evolution of the secondary. After an integration time of only $30 \mathrm{~d}$, there is a spread of $\sim 15^{\circ}$ in the relative positions of the secondaries. The DART Investigation Team has a requirement to predict the orbit phase at the impact epoch to within $\pm 45^{\circ} 60$ days prior to launch and to within $\pm 15^{\circ} 55$ days prior to impact ( $3 \sigma$ errors). The high sensitivity to Didymos A's initial rotation phase means that it will likely be impossible to meet this requirement with dynamical simulations, especially considering the uncertainties in the other initial conditions. However, the Observing Working Group should be able to meet this orbital phase prediction requirement through 


\section{Orbit Phase vs. Initial Primary Rotation Phase}

All quantitites are relative to their respective nominal run

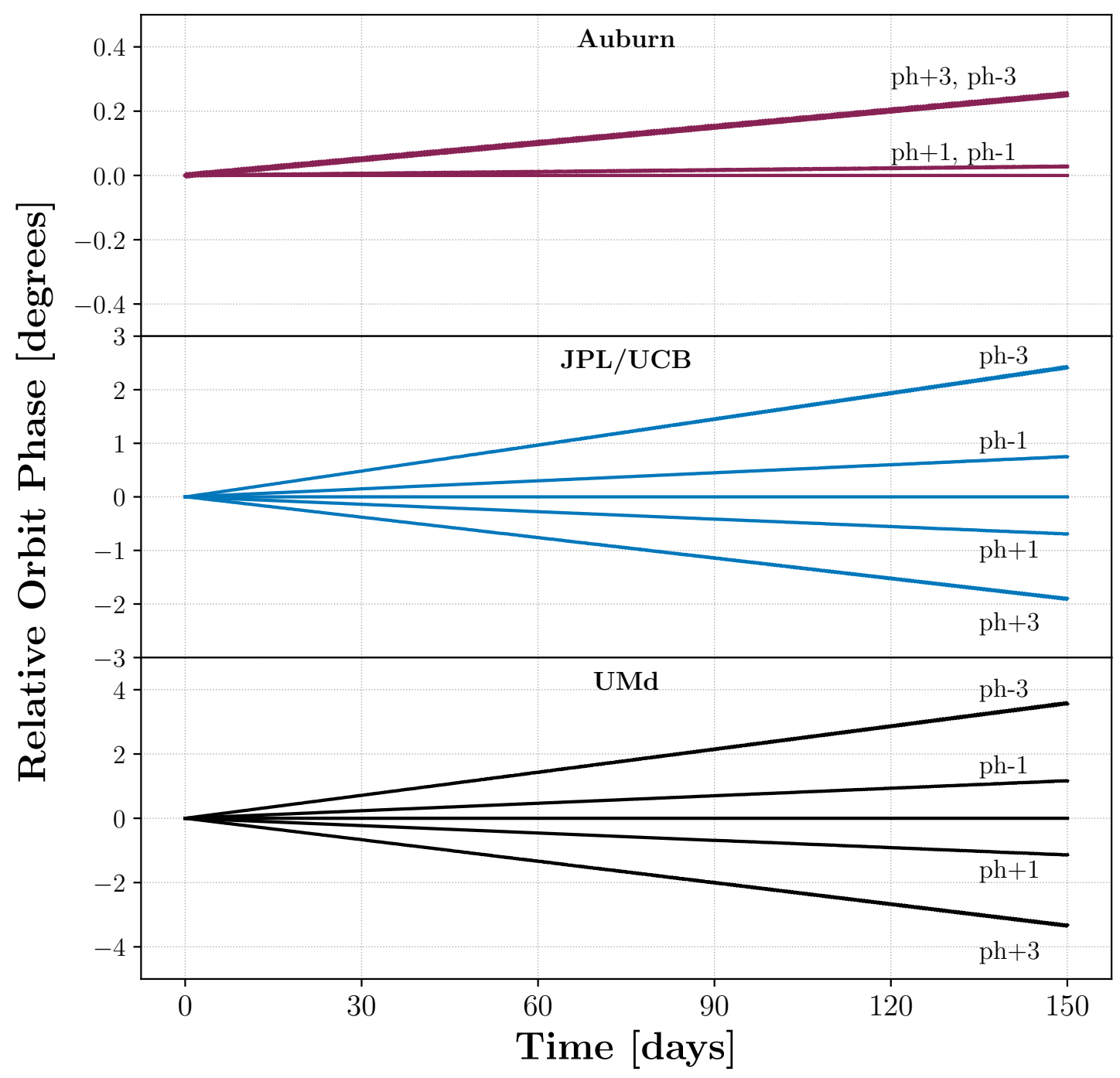

Figure 5: Orbit phase relative to each code's nominal run (the horizontal line). Small differences in the initial primary rotation phase give a slightly different orbital period, making it difficult to predict the position of the secondary at later times. Note the differences in vertical scale between codes. 


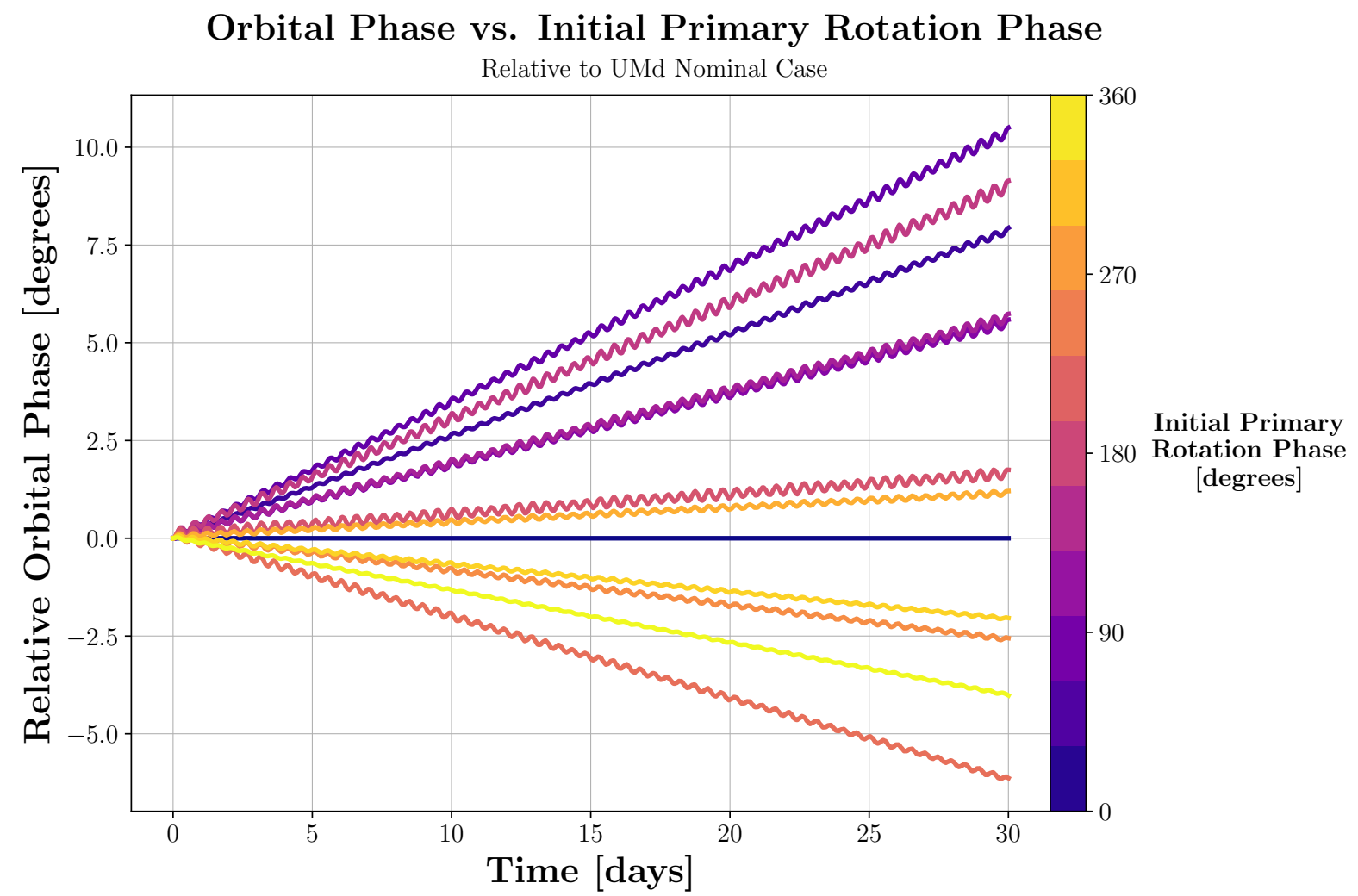

Figure 6: Orbit phase relative to nominal run, for initial primary rotation phases spanning $360^{\circ}$, using the UMd code. This represents the spread in the possible locations of Didymos B after a fixed interval of time given some random initial primary rotation phase. In order to distinguish each curve, this figure is best viewed in the online color version.

fitting a weighted least-squares model to observed timing of mutual events (Naidu et al., 2020b).

\subsection{Didymos B Libration}

Through tidal dissipation, we expect that the mutual orbit has circularized, the secondary is tidally locked, and any libration of the secondary's spin state has damped to a minimum. So the Didymos system should be in or close to a dynamically relaxed state prior to the DART impact. The impact will nearly instantaneously reduce the instantaneous orbital velocity of the second-ary, decreasing the orbit period and increasing the eccentricity. Therefore, significant libration of the secondary should be induced. A libration angle is a measure of the orientation of a satellite's long axis relative to the line of centers between the two components' centers of mass. In the following analysis, we show only results from the UCB 4th order code for brevity, although we note that all 4 codes show good agreement, given the known differences among the codes. 
In the coupled spin-orbit problem in which a synchronous, ellipsoidal secondary orbits a spherical or point-mass primary with its spin axis aligned with the mutual orbit pole, there are two modes of libration: excited and relaxed. In the decoupled spin-orbit problem, the excited and relaxed modes are analogous to free and forced librations, respectively. See Naidu and Margot (2015) for a detailed discussion on these two libration modes in both the coupled and uncoupled scenarios.

The frequency of free libration for a synchronous satellite on a circular orbit is given by,

$$
\omega_{0}=n\left(3 \frac{\mathcal{B}-\mathcal{A}}{C}\right)^{1 / 2},
$$

where $n$ is the mean motion, and $\mathcal{A}, \mathcal{B}$, and $\mathcal{C}$ are the three principal moments of inertia of the secondary, where $\mathcal{A}<\mathcal{B}<C$ (Murray and Dermott, 2000). This mode is analogous to a pendulum's natural frequency, depending on its length and the gravitational acceleration. This libration mode is thought to be damped away due to tidal friction, especially if the secondary has a rubble-pile structure (Murray and Dermott, 2000; Goldreich and Sari, 2009). However, the forced (relaxed) mode necessarily exists for a synchronous secondary on an eccentric orbit. The secondary will feel a periodic restoring torque, with a frequency equal to the mean motion, due to the misalignment of the long axis with the line of centers, resulting from the orbital angular velocity varying over the course of a single orbit.

This picture is complicated when we consider libration in the full two-body problem. The theory on spin-orbit coupling discussed above makes two critical assumptions: 1) that the orbit is fixed (no apsis precession) and 2) that the ellipsoidal secondary is orbiting a spherically symmetric primary (Wisdom, 1987). As a result, we will see some differences between the classic theory and our simulation results.

The nominal DART spacecraft impact is designed to hit the secondary's center-of-figure, in a direction nearly opposite its orbital motion at a 15-to-25-degree angle with respect to the orbital plane (depending on DART's trajectory), imparting a near-instantaneous change to its orbital velocity without significantly altering its spin state(Cheng et al., 2018). This will induce both longitudinal (in-plane) and latitudinal (out-of-plane) librations that will have both relaxed and excited 
components. By changing the mean motion without a matching change in the secondary's spin rate, we introduce excited (free) libration modes on top of those that exist already (if any). Further, the impact will increase the eccentricity of the system, exciting a stronger relaxed (forced) libration mode. Therefore, studying the resulting libration for the benchmarking cases where we perturb the orbital motion of the secondary reveals the extent to which DART may effect a libration in the secondary. Further, understanding the behavior of induced librations may be an important tool for interpreting the results of the DART impact, if the libration amplitude or frequency is observable.

The longitudinal libration for the nominal case is shown in Fig. 7a for the first 10 days of the simulation. The distinct beating pattern in the libration is a signature of both excited-mode and relaxed-mode librations (Naidu and Margot, 2015). A Fourier transform of the libration pattern shows the two distinct libration modes (Fig. 7b).

The frequency of the relaxed (forced) mode is the frequency at which the orbital angular velocity oscillates, which in this case is the epicyclic or radial frequency. A key assumption in the classic spin-orbit problem is that the orbit is fixed (i.e., no apsidal precession), in which case the epicyclic frequency would match the mean motion. However, the oblate shape of the primary and the close orbit of the secondary results in an extremely fast precession of the periapse. In the nominal case, the mean motion differs from the epicyclic frequency by $\sim 1 \%$, which corresponds to a precession rate of $\sim 3.5^{\circ}$ per orbit.

The excited (free) libration mode has a frequency close to the theoretical prediction given by Eq. 1. These frequencies don't match perfectly because Eq. 1 assumes a spherically symmetric primary on a fixed orbit. Because this excited libration frequency will depend on the secondary's moments of inertia in a fashion similar to Eq. 1, it may be possible to infer something about the mass distribution and interior structure from a careful measurement of the libration frequency with Hera.

Fig. 8 shows the libration for the nominal case and the 2 cases where the secondary was given an along-track velocity perturbation (posT/negT). The libration amplitude is driven by the initial difference between the orbital angular velocity and the secondary's spin rate. The posT case is where the secondary is given a slightly larger initial tangential velocity, so its orbit expands, increasing the orbital period to $\sim 11.911 \mathrm{~h}$, closely matching Didymos B's initial spin period and 


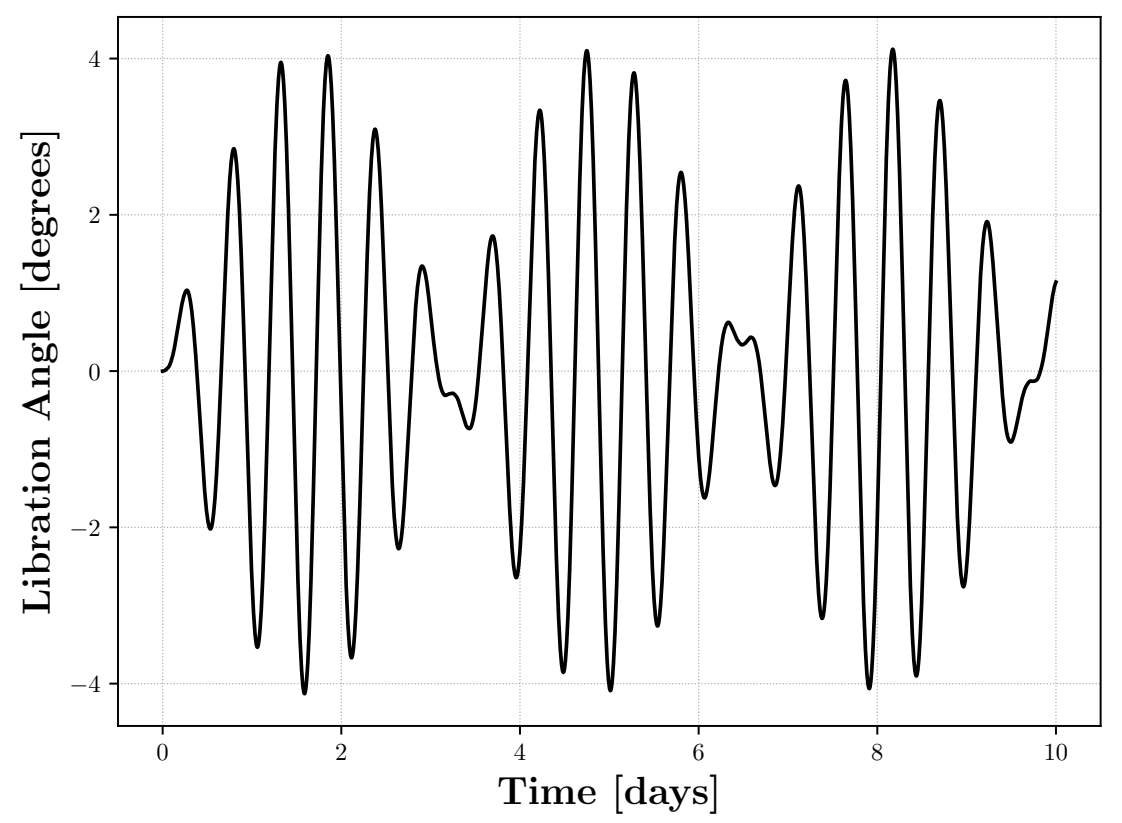

(a) Longitudinal libration of UCB nominal case over 10 days. The libration pattern is consistent over the full 150-day simulation.

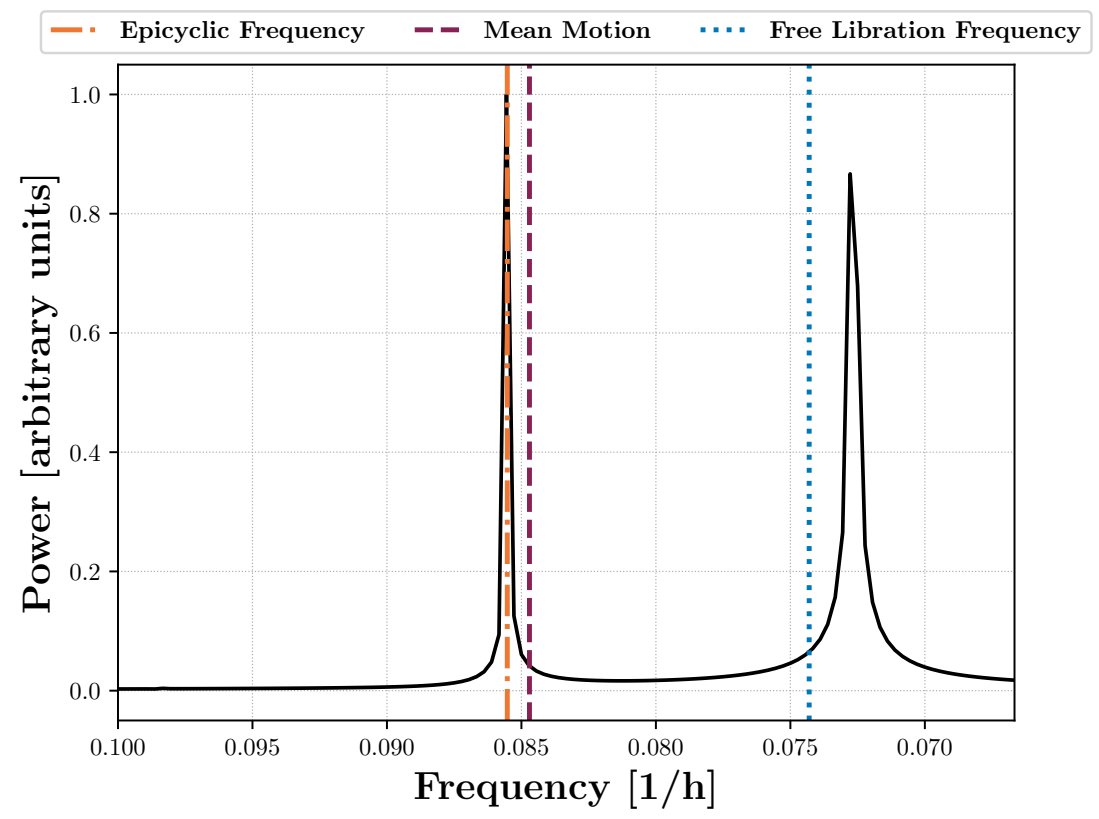

(b) Fourier transform of longitudinal libration.

Figure 7: The Fourier transform of the longitudinal libration reveals the two libration modes. The relaxed (forced) mode is driven by the epicyclic (radial) frequency, while the excited (free) mode is controlled by the moments of inertia of the secondary. The theoretical free libration frequency doesn't match the excited mode perfectly because its derivation assumes a spherically symmetric primary. 
thus decreasing the libration amplitude. The negT case is the opposite: a smaller initial tangential velocity shrinks the orbit and shortens the orbital period $(\sim 11.703 \mathrm{~h})$, producing a larger discrepancy between the secondary's initial spin and orbital periods, thus a libration amplitude reaching $\sim 8^{\circ}$ at its maximum.

The perturbation to the secondary's linear momentum in the posT and negT cases is approximately one-half of the momentum carried by the DART spacecraft, so these perturbations are of the same order of magnitude that DART may produce. Since the posT case has a relatively small libration amplitude, we can think of it as being close to the "true" relaxed state of the system (in which the excited libration mode has nearly damped away but relaxed librations persist). Then, the jump from the posT to the negT cases corresponds to a rough conservative estimate of the effect of the DART impact on the libration, when the momentum perturbation to the secondary is approximately equal to the momentum carried by the DART spacecraft. In reality, we would expect the momentum perturbation to the secondary to be considerably larger, due to the contributions of ejecta to the net momentum transfer. Further, if the DART spacecraft impacts several meters off of center-of-figure, which terminal guidance simulations at JHU/APL suggest is likely, the torque applied to the secondary will also alter its spin state, nearly instantaneously. The DART terminal guidance system will likely result in an impact location biased toward the illuminated portion of the secondary, which will be the side opposite the primary based on DART's viewing geometry at the impact epoch. Therefore, such an off-center impact is likely to reduce the secondary's angular velocity, further increasing the maximum possible libration amplitude. For these reasons, the simulations presented here are a conservative estimate of the possible post-impact libration state of Didymos B, given our current knowledge of the state of the system. It is also important to note, that the libration amplitude and frequency are dependent on Didymos B's moments of inertia which are computed based on our assumptions of constant density and its ellipsoidal shape. Studying the dependence of Didymos B's libration on its mass distribution is planned for a future study.

Naidu and Margot (2015) show that the libration of a synchronous satellite may be detectable with radar, if the secondary is large enough compared to the primary $\left(D_{S} / D_{P} \gtrsim 0.2\right)$. This threshold is barely satisfied by the Didymos system and therefore the libration may be measurable with 


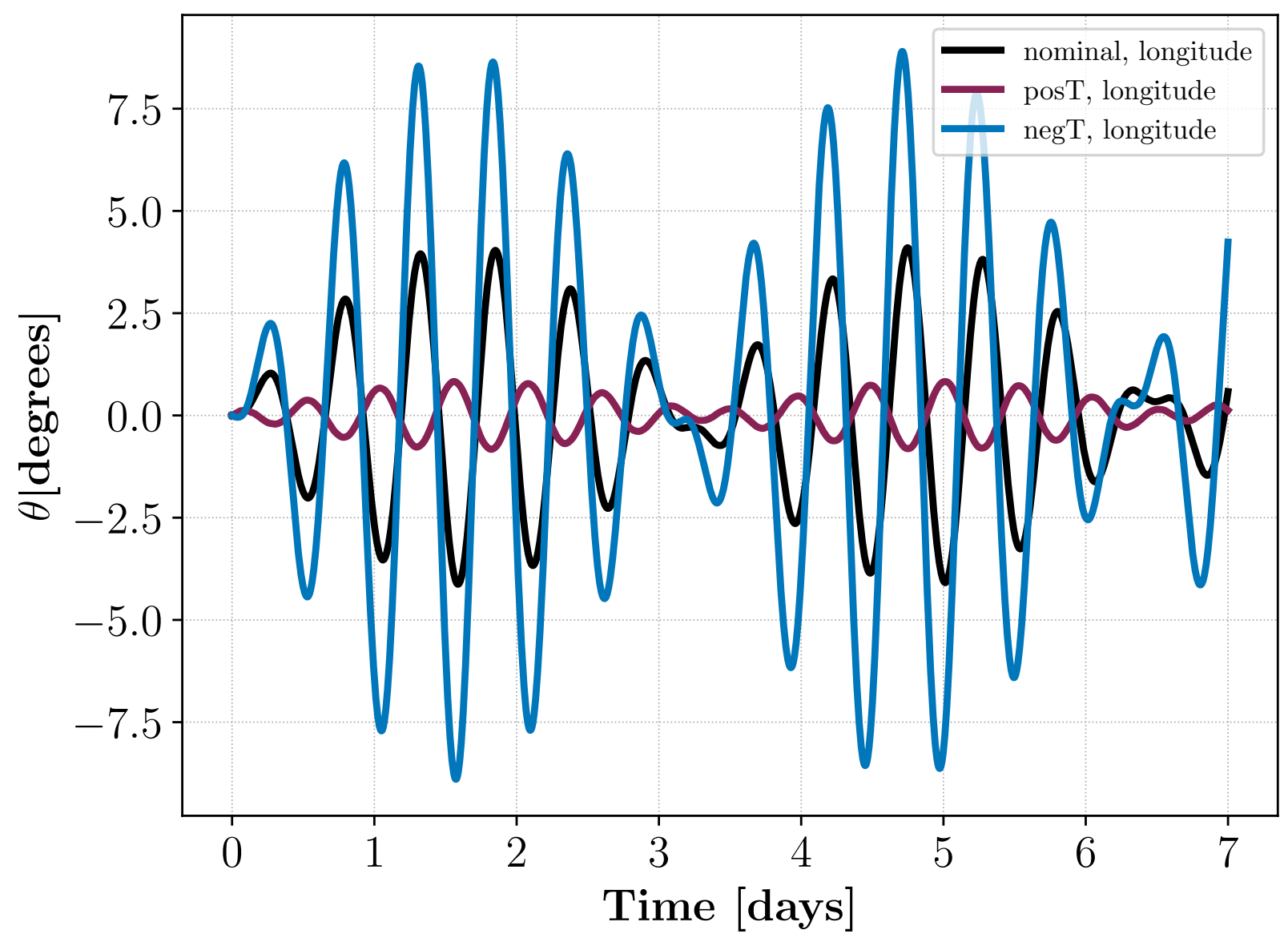

Figure 8: Longitudinal libration angles a function of time for the nominal run and tangential (along-track) perturbations, using the UCB code. In order to distinguish each curve, this figure is best viewed in the online color version.

radar, given adequate observing conditions, a favorably shaped secondary, and a sufficiently large momentum transfer.

When the secondary is given a normal (out-of-plane) perturbation, we are inducing out-ofplane motion in the secondary and therefore a noticeable, but small, latitudinal libration in the secondary, while the longitudinal libration is effectively unchanged (see Fig. 9). The librations resulting from the benchmarking cases where we apply radial velocity perturbations (posR/negR) and different primary rotation phases $(\mathrm{ph} \pm 3$ and $\mathrm{ph} \pm 1)$ show almost no sensitivity, so we exclude showing them here. 


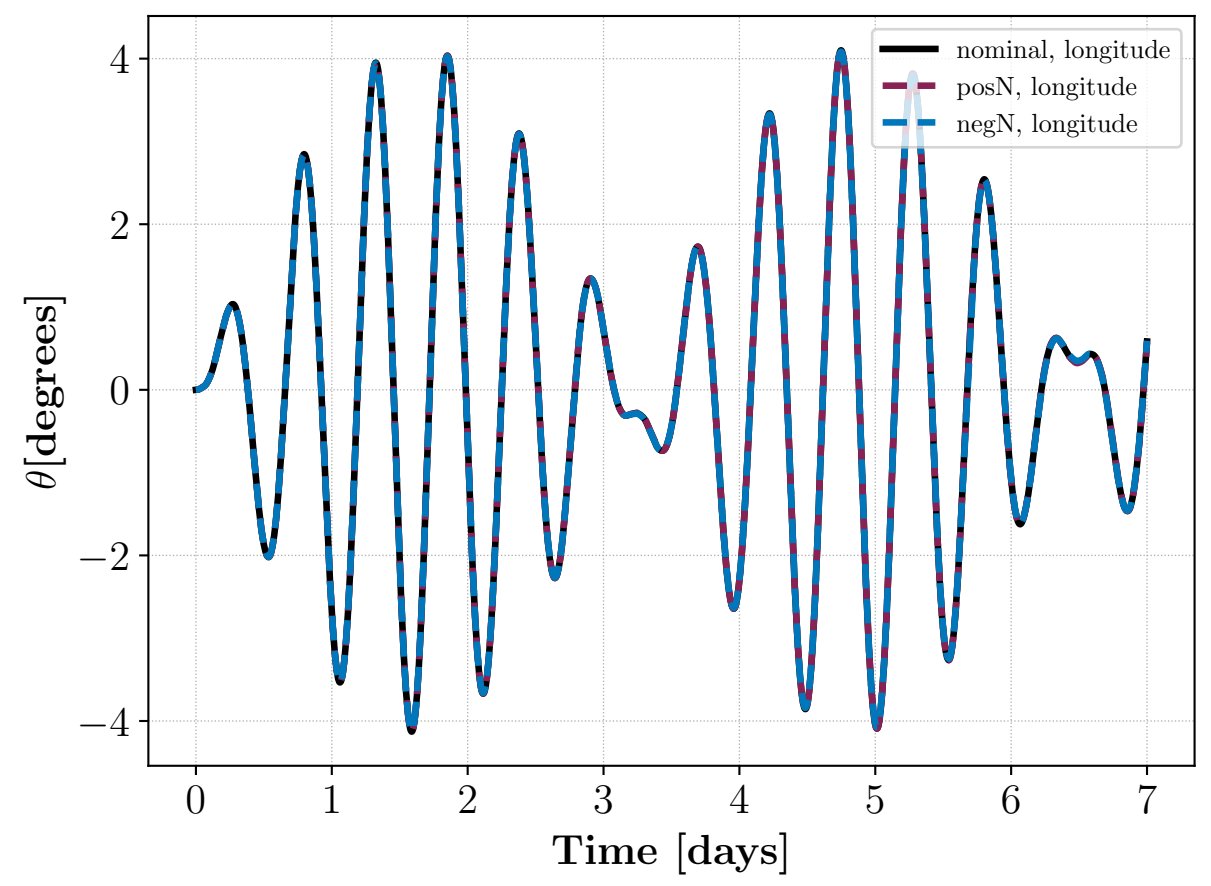

(a) Longitudinal libration for out-of-plane perturbations.

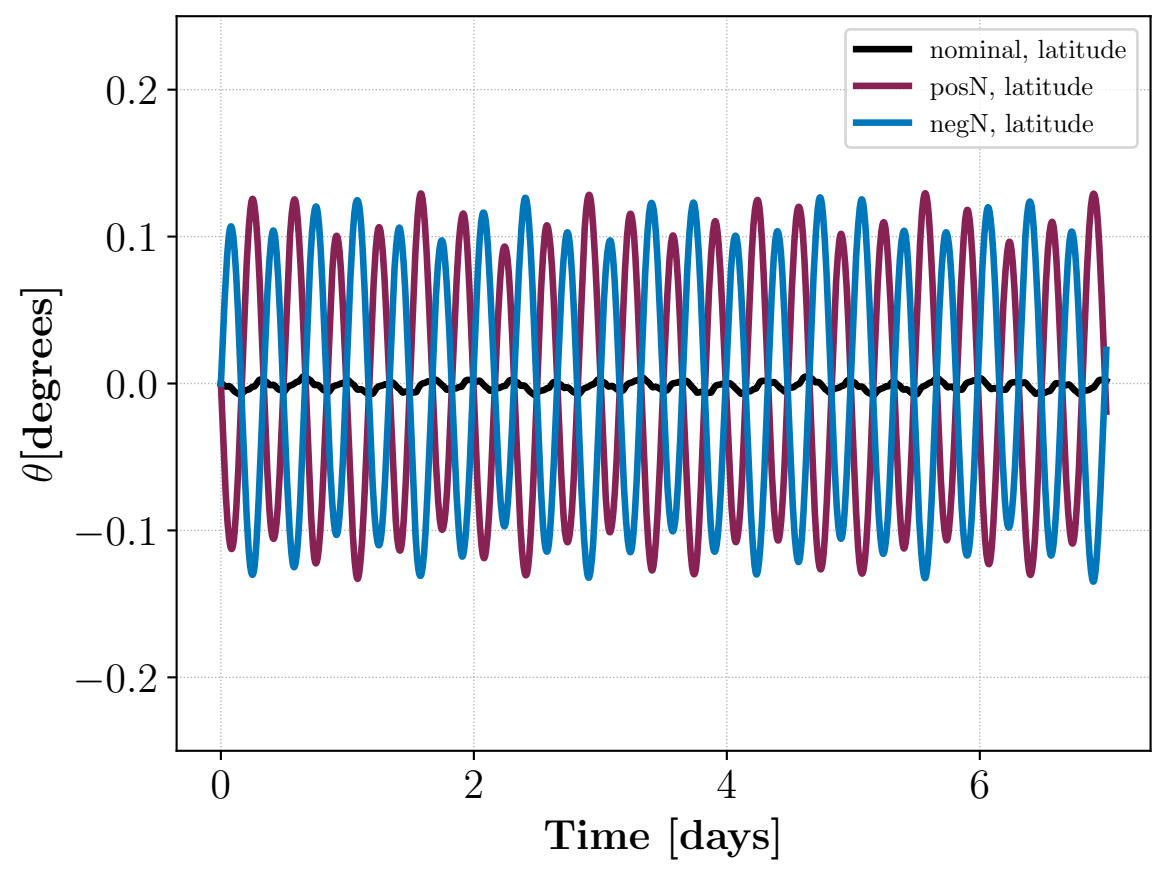

(b) Latitudinal libration for out-of-plane perturbations.

Figure 9: Longitudinal and latitudinal libration vs. time for normal (out-of-plane) perturbations, using the UCB code. The longitudinal libration is insensitive to the normal perturbation as expected, while the small induced latitudinal libration is caused by initial out-of-plane motion of the secondary. In order to distinguish each curve, this figure is best viewed in the online color version. 


\section{Conclusions}

In this work, we found that the simulation package provided by UC Boulder is well-suited to studying the orbital dynamics of the Didymos system, due to its accuracy and speed. The Dynamics Working Group has recommended the adoption of this code for future dynamics studies in support of DART.

The results of this benchmarking study show that: 1) shape perturbations cause a non-negligible deviation from a Keplerian orbit; 2) the orbit phase of the secondary is highly dependent on the initial orientation of the primary; and 3) the system will be highly susceptible to induced librations resulting from the DART impact, which may be measurable from ground-based radar or with Hera. If measurable, Didymos B's libration may be a useful probe of its internal structure.

The first two results indicate that predicting the orbital phase of the secondary may not be feasible with numerical simulations, given the uncertainties in the initial conditions and body shapes. However, the Observing Working Group will be able to meet this orbital phase prediction requirement through fitting an analytic model to observed timing of mutual events.

We have begun a comprehensive study with the UCB code on the strength and frequency of post-impact librations as a function of the mass distribution of Didymos B and momentum transferred by DART. This study will be used to constrain the range of possible impact outcomes in order to better infer the result of the actual DART experiment.

In reality, Didymos $\mathrm{A}$ is likely a rubble pile given its shape and fast rotation. A rubble-pile structure may play an important role for the binary dynamics due to processes such as landslides (Hirabayashi et al., 2019) and tidal dissipation. Didymos B may also be a rubble pile in which case its free libration modes will dampen via internal friction. Therefore, we also plan to use PKDGRAV with an implementation of a soft-sphere discrete element method to numerically investigate whether this damping may be a noticeable effect over the timescales between the DART and Hera missions. 


\section{Acknowledgments}

This study was supported in part by the DART mission, NASA Contract \# NNN06AA01C to JHU/APL. Some of this research was carried out at the Jet Propulsion Laboratory, California Institute of Technology, under a contract with the National Aeronautics and Space Administration (80NM0018D0004).

H.F.A. would like to thank Doug Hamilton for useful discussions. A.B.D. was supported by the National Science Foundation Graduate Research Fellowship Program under Grant No. DGE 1650115. Any opinions, findings, and conclusions or recommendations expressed in this material are those of the author(s) and do not necessarily reflect the views of the National Science Foundation. M.H. acknowledges the Auburn University intramural grant program support. P.M. acknowledges the European Space Agency (ESA) and the French Space Agency (CNES) for support.

Some of the simulations herein were carried out on: 1) The University of Maryland Astronomy Department's YORP cluster, administered by the Center for Theory and Computation; 2) the Stampede 2 cluster at the Texas Advanced Computing Center (TACC), University of Texas at Austin, funded by NSF award ACI-1134872; and 3) the nexus cluster of the Mission Design and Navigation Section at JPL. Raytracing for Fig. 1b was performed using the Persistence of Vision Raytracer (http://povray.org/).

\section{References}

Alday, A., Moore, K., Tranilla, M., Goggia, T., Razo, V., Sylva, A., Ota, M., Fricke, G., Becker, J., Africano, J., Sydney, P., Nishimoto, D., O’Connell, D., Kervin, P., Kraszewski, B., Soo Hoo, V., Montani, J., Scotti, J.V., Williams, G.V., 1996. 1996 GT. Minor Planet Electronic Circulars 1996-H02.

Cheng, A.F., Rivkin, A.S., Michel, P., Atchison, J., Barnouin, O., Benner, L., Chabot, N.L., Ernst, C., Fahnestock, E.G., Kueppers, M., Pravec, P., Rainey, E., Richardson, D.C., Stickle, A.M., Thomas, C., 2018. AIDA DART asteroid deflection test: Planetary defense and science objectives. Planet. Space Sci. 157, 104-115.

Goldreich, P., Sari, R., 2009. Tidal evolution of rubble piles. The Astrophysical Journal 691, 54-60.

Hirabayashi, M., Davis, A.B., Fahnestock, E.G., Richardson, D.C., Michel, P., Cheng, A.F., Rivkin, A.S., Scheeres, D.J., Chesley, S.R., Yu, Y., Naidu, S.P., Schwartz, S.R., Benner, L.A.M., Pravec, P., Stickle, A.M., Jutzi, M., 
2019. Assessing possible mutual orbit period change by shape deformation of Didymos after a kinetic impact in the NASA-led Double Asteroid Redirection Test. Advances in Space Research 63, 2515-2534.

Hirabayashi, M., Scheeres, D.J., 2013. Recursive computation of mutual potential between two polyhedra. Celestial Mechanics and Dynamical Astronomy 117, 245-262.

Hou, X., Scheeres, D.J., Xin, X., 2017. Mutual potential between two rigid bodies with arbitrary shapes and mass distributions. Celestial Mechanics and Dynamical Astronomy 127, 369-395.

Lee, T., Leok, M., McClamroch, N.H., 2007. Lie group variational integrators for the full body problem in orbital mechanics. Celestial Mechanics and Dynamical Astronomy 98, 121-144.

Michel, P., Kueppers, M., Sierks, H., Carnelli, I., Cheng, A.F., Mellab, K., Granvik, M., Kestilä, A., Kohout, T., Muinonen, K., Näsilä, A., Penttila, A., Tikka, T., Tortora, P., Ciarletti, V., Hérique, A., Murdoch, N., Asphaug, E., Rivkin, A., Barnouin, O., Bagatin, A.C., Pravec, P., Richardson, D.C., Schwartz, S.R., Tsiganis, K., Ulamec, S., Karatekin, O., 2018. European component of the AIDA mission to a binary asteroid: Characterization and interpretation of the impact of the DART mission. Advances in Space Research 62, 2261-2272.

Murray, C.D., Dermott, S.F., 2000. Solar System Dynamics. Cambridge University Press.

Naidu, S., Benner, L., Brozovic, M., Nolan, M., Ostro, S., Margot, J., Giorgini, J., Hirabayashi, T., Scheeres, D., Pravec, P., Scheirich, P., Magri, C., Jao, J., 2020a. Radar observations and a physical model of binary near-earth asteroid 65803 didymos, target of the dart mission. Icarus , 113777.

Naidu, S., Chesley, S., Farnocchia, D., 2020b. Computation of the orbit of the satellite of binary near-earth asteroid (65803) didymos. Jet Propulsion Laboratory Interoffice Memorandum 392R-20-001.

Naidu, S.P., Margot, J.L., 2015. Near-earth asteroid satellite spins under spin-orbit coupling. Astronomical Journal $149,80$.

Pravec, P., Benner, L.A.M., Nolan, M.C., Kusnirak, P., Pray, D., Giorgini, J.D., Jurgens, R.F., Ostro, S.J., Margot, J.L., Magri, C., Grauer, A., Larson, S., 2003. (65803) 1996 GT. IAU Circ. 8244, 2.

Pravec, P., Scheirich, P., Kušnirák, P., Šarounová, L., Mottola, S., Hahn, G., Brown, P., Esquerdo, G., Kaiser, N., Krzeminski, Z., Pray, D.P., Warner, B.D., Harris, A.W., Nolan, M.C., Howell, E.S., Benner, L.A.M., Margot, J.L., Galád, A., Holliday, W., Hicks, M.D., Krugly, Y.N., Tholen, D., Whiteley, R., Marchis, F., DeGraff, D.R., Grauer, A., Larson, S., Velichko, F.P., Cooney, W.R., Stephens, R., Zhu, J., Kirsch, K., Dyvig, R., Snyder, L., Reddy, V., Moore, S., Gajdoš, Š., Világi, J., Masi, G., Higgins, D., Funkhouser, G., Knight, B., Slivan, S., Behrend, R., Grenon, M., Burki, G., Roy, R., Demeautis, C., Matter, D., Waelchli, N., Revaz, Y., Klotz, A., Rieugné, M., Thierry, P., Cotrez, V., Brunetto, L., Kober, G., 2006. Photometric survey of binary near-Earth asteroids. Icarus $181,63-93$.

Richardson, D.C., Michel, P., Walsh, K., Flynn, K., 2009. Numerical simulations of asteroids modelled as gravitational aggregates with cohesion. Planetary and Space Science 57, 183 - 192. Catastrophic Disruption in the Solar System. Richardson, D.C., Quinn, T., Stadel, J., Lake, G., 2000. Direct Large-Scale N-Body Simulations of Planetesimal 
Dynamics. Icarus 143, 45-59.

Scheirich, P., Pravec, P., 2009. Modeling of lightcurves of binary asteroids. Icarus 200, 531-547.

Stadel, J.G., 2001. Cosmological N-body simulations and their analysis. Ph.D. thesis. University of Washington.

Werner, R.A., Scheeres, D.J., 2005. Mutual Potential of Homogeneous Polyhedra. Celestial Mechanics and Dynamical Astronomy 91, 337-349.

Wisdom, J., 1987. Urey prize lecture: Chaotic dynamics in the solar system. Icarus 72, $241-275$. 